\section{Gender-specific outcomes of cardiac resynchronisation therapy with or without defibrillator}

\author{
Valentina Kutyifa
}

Cardiac resynchronisation therapy has been shown to significantly reduce heart failure (HF) symptoms, prevent HF hospitalisations and improve survival in $\mathrm{HF}$ patients with severely reduced left ventricular ejection fraction (LVEF) and a wide QRS. ${ }^{1}$ Sudden cardiac death (SCD) is also prevalent in $\mathrm{HF}$ patients with severely reduced LVEF, and therefore, combined protection from HF and SCD is generally warranted in this cohort. It is however not well known whether adding a defibrillator function to cardiac resynchronisation therapy provides incremental benefit, especially in the context of gender.

In their Heart publication Barra et al. ${ }^{2}$ reported retrospective analyses of outcomes of 5307 consecutive patients with ischaemic or non-ischaemic cardiomyopathy and no history of sustained ventricular tachyarrhythmia implanted either with cardiac resynchronisation therapy with defibrillator (CRT-D) or without a defibrillator (CRT-P). The primary focus of their report was to assess incremental survival benefit of CRT-D versus CRT-P by gender. Using propensity score matching, they showed no survival advantage of CRT-D versus CRT-P in men or women. Using inverse probability weighting analysis, a benefit of CRT-D over CRT-P was present in men $(\mathrm{HR}=0.78$, $95 \%$ CI 0.65 to $0.94, p=0.012)$ but not in men $(\mathrm{HR}=0.87,95 \% \mathrm{CI} 0.63$ to 1.19 , $\mathrm{p}=0.043)$. The excess mortality was less likely related to SCD in women than in men.

The study by Barra et al raises an important clinical question and provides valuable information on outcomes of CRT-D versus CRT-P by gender, in a large multicentre cohort. The findings are intriguing and present further considerations for everyday clinical practice.

What are the most important takehome messages of this publication? First, the authors have shown that patients with an implanted CRT-P are older, they have more advanced HF and more

\footnotetext{
Correspondence to Dr Valentina Kutyifa, Heart Research Follow-up Program, Cardiology Division, University of Rochester Medical Center, 265 Crittenden Blvd., Rochester, NY 14642, USA; valentina.kutyifa@ heart.rochester.edu
}

comorbidities. Interestingly, there were $42 \%$ women in the CRT-P group compared with $15 \%$ in the CRT-D group $(\mathrm{p}<0.001)$, suggesting that gender alone might have contributed to device selection. Importantly, this study again shows that women are under-represented among CRT recipients. While we have been increasingly enrolling women in our Multicenter Automatic Defibrillator Implantation Trials (MADIT), ${ }^{1}$ further efforts are nevertheless needed to close this gap. One such effort is the Women Opt In for Heart Research initiative, aiming to increase female enrolment in the Multicenter Automatic Defibrillator Implantation Trial with the Subcutaneous implantable cardioverter defibrillator (ICD) (MADIT S-ICD), ${ }^{3}$ and in the Assessment of the WATCHMAN Device in Patients Unsuitable for Oral Anticoagulation study. Since patients were so different in the two subgroups of this study, multiple statistical approaches were appropriately used for the assessment of CRT-D versus CRT-P effects on mortality. Such heterogeneity can be mitigated using selected statistical approaches, however, definitive answer on whether CRT-P provides similar survival benefit in women to CRT-D could only come from prospective, randomised, clinical trials.

Second, the authors elegantly show differential outcomes of CRT-D versus CRT-P in men and women. Not only they suggest that women do not have an incremental survival benefit from CRT-D over CRT-P, they also report that men at higher risk for competing non-cardiac death do not benefit from CRT-D. Such observations underscore the importance of personalised medicine, an approach that goes beyond assessing a single covariate to determine response or outcomes. It is conceivable that proper patient selection for CRT-D versus CRT-P requires assessment of multiple factors such as baseline demographics, heart disease aetiology and severity, history of HF and specific prior treatment modalities. Newer data suggest that genetic information might also serve as a powerful surrogate for susceptibility of ventricular arrhythmias and potentially, eligibility for a defibrillator. ${ }^{4}$ And when assessing indication for CRT-D versus
CRT-P, not only potential benefits, but also risks have to be taken into consideration. Device-related complications, infections and inappropriate ICD shocks are major issues related to CRT-D implantations that are differently affecting women and men, ${ }^{5}$ and therefore, individual risk-benefit assessment is warranted.

Third, the study provides important information on the cause of death, showing that the excess mortality of CRT-P patients compared with CRT-D was related to SCD in $7.4 \%$ of the cases in men, but only in $2.2 \%$ in women. It is important to mention that this analysis was not adjusted for differences in clinical characteristics. Rates of SCD were lowest among female patients with non-ischaemic cardiomyopathy, irrespective of implanted CRT-D versus CRT-P. This could be the key message of the present report. Aetiology of cardiomyopathy might be a relevant confounder and effect modifier in this analysis. Previous report suggested benefit of CRT-D over CRT-P in patients with ischaemic cardiomyopathy, but not in patients with non-ischaemic cardiomyopathy. ${ }^{6}$ It has been well described that female patients implanted with CRT devices more often present with non-ischaemic cardiomyopathy and derive greater improvement in echo outcomes and clinical outcomes than patients with ischaemic cardiomyopathy. ${ }^{7}$ It has also been shown that improvement in clinical symptoms and echocardiography parameters are accompanied by reduction in ventricular arrhythmia risk. ${ }^{8}$ Thus, it is conceivable that women who get more benefit from CRT-P even without a defibrillator, exhibit such a low risk of arrhythmias that CRT-D is no longer able to provide incremental benefit over CRT-P.

The question raised by Barra et al is important, and the authors are to be congratulated on their relevant analyses. This study adds to the growing body of literature on the effectiveness of CRT-D versus CRT-P in HF patients. However, given the non-randomised nature of this observation, selection bias, potential unmeasured confounders and the relatively short follow-up (about 3 years), further, preferably prospective, well-designed randomised studies are needed.

In summary, the study by Barra et al suggests differential sex-specific outcomes of cardiac resynchronisation therapy with or without a defibrillator, with only selected male patients deriving incremental survival benefit from cardiac resynchronisation therapy with a defibrillator. 
Contributors I have drafted this article.

Competing interests None declared.

Provenance and peer review Commissioned; internally peer reviewed.

(c) Article author(s) (or their employer(s) unless otherwise stated in the text of the article) 2017. All rights reserved. No commercial use is permitted unless otherwise expressly granted.

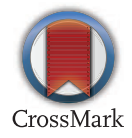

To cite Kutyifa V. Heart 2017;103:732-733.

Published Online First 10 March 2017

\section{SLinked}

http://dx.doi.org/10.1136/heartjnl-2016-310677
Heart 2017;103:732-733.

doi:10.1136/heartjnl-2016-311024

\section{REFERENCES}

1 Moss AJ, Hall WJ, Cannom DS, et al; MADIT-CRT Trial Investigators. Cardiac-resynchronization therapy for the prevention of heart-failure events. $N$ Eng/ J Med 2009;361:1329-38.

2 Barra S, Providência R, Duehmke R, et al. Sexspecific outcomes with addition of defibrillation to resynchronisation therapy in patients with heart failure. Heart 2017;103:753-60

3 National Institutes of Health. Multicenter automatic defibrillator implantation trial with subcutaneous implantable cardioverter defibrillator (MADIT S-ICD). https://clinicaltrials.gov/ct2/show/ NCT02787785.

4 Jabbari R, Glinge C, Jabbari J, et al. A common variant in SCN5A and the risk of ventricular fibrillation caused by first ST-Segment elevation myocardial infarction. PLoS One 2017;12:e0170193.
5 Jamerson D, McNitt S, Polonsky S, et al. Early procedure-related adverse events by gender in MADIT-CRT. J Cardiovasc Electrophysiol 2014:25:985-9.

6 Kutyifa V, Geller L, Bogyi P, et al. Effect of cardiac resynchronization therapy with implantable cardioverter defibrillator versus cardiac resynchronization therapy with pacemaker on mortality in heart failure patients: results of a highvolume, single-centre experience. Eur I Heart Fail 2014;16:1323-30.

7 Arshad A, Moss AJ, Foster E, et al; MADIT-CRT Executive Committee. Cardiac resynchronization therapy is more effective in women than in men: the MADIT-CRT (Multicenter automatic defibrillator implantation trial with cardiac resynchronization therapy) trial. J Am Coll Cardiol 2011;57:813-20.

8 Barsheshet A, Wang PJ, Moss AJ, et al. Reverse remodeling and the risk of ventricular tachyarrhythmias in the MADIT-CRT (Multicenter automatic defibrillator implantation trial-cardiac resynchronization therapy). J Am Coll Cardiol 2011;57:2416-23. 